\title{
Multi-hop Attention Graph Neural Networks
}

\author{
Guangtao Wang $^{1 *}$, Rex Ying ${ }^{2}$, Jing Huang ${ }^{1}$ and Jure Leskovec ${ }^{2}$ \\ ${ }^{1}$ JD AI Research \\ ${ }^{2}$ Computer Science, Stanford University \\ guangtao.wang@jd.com, rexying@stanford.edu,jing.huang@jd.com, jure@cs.stanford.edu
}

\begin{abstract}
Self-attention mechanism in graph neural networks (GNNs) led to state-of-the-art performance on many graph representation learning tasks. Currently, at every layer, attention is computed between connected pairs of nodes and depends solely on the representation of the two nodes. However, such attention mechanism does not account for nodes that are not directly connected but provide important network context. Here we propose Multi-hop Attention Graph Neural Network (MAGNA), a principled way to incorporate multihop context information into every layer of attention computation. MAGNA diffuses the attention scores across the network, which increases the "receptive field" for every layer of the GNN. Unlike previous approaches, MAGNA uses a diffusion prior on attention values, to efficiently account for all paths between the pair of disconnected nodes. We demonstrate in theory and experiments that MAGNA captures large-scale structural information in every layer, and has a low-pass effect that eliminates noisy high-frequency information from graph data. Experimental results on node classification as well as the knowledge graph completion benchmarks show that MAGNA achieves state-ofthe-art results: MAGNA achieves up to $5.7 \%$ relative error reduction over the previous state-of-theart on Cora, Citeseer, and Pubmed. MAGNA also obtains the best performance on a large-scale Open Graph Benchmark dataset. On knowledge graph completion MAGNA advances state-of-the-art on WN18RR and FB15k-237 across four different performance metrics.
\end{abstract}

\section{Introduction}

Self-attention [Bahdanau et al., 2015; Vaswani et al., 2017] has pushed the state-of-the-art in many domains including graph presentation learning [Devlin et al., 2019]. Graph Attention Network (GAT) [Veličković et al., 2018] and related models [Li et al., 2018; Wang et al., 2019a; Liu et al., 2019;

\footnotetext{
${ }^{*}$ Contact Author: xjtuwgt@gmail.com
}
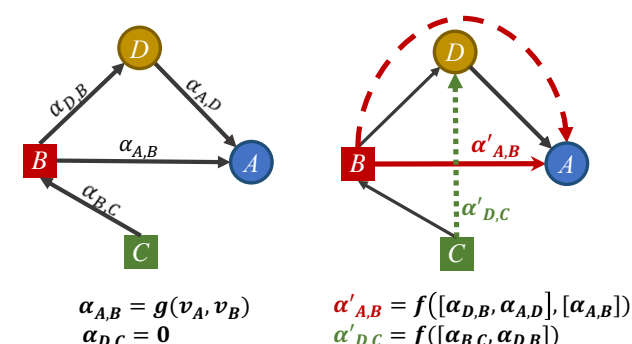

Figure 1: Multi-hop attention diffusion. Consider making a prediction at nodes $A$ and $D$. Left: A single GAT layer computes attention scores $\boldsymbol{\alpha}$ between directly connected pairs of nodes (i.e., edges) and thus $\boldsymbol{\alpha}_{D, C}=0$. Furthermore, the attention $\boldsymbol{\alpha}_{A, B}$ between $A$ and $B$ only depends on node representations of $A$ and $B$. Right: A single MAGNA layer: (1) captures the information of $D$ 's two-hop neighbor node $C$ via multi-hop attention $\boldsymbol{\alpha}^{\prime}{ }_{D, C}$; and (2) enhances graph structure learning by considering all paths between nodes via diffused attention, which is based on powers of graph adjacency matrix. MAGNA makes use of node $D$ 's features for attention computation between $A$ and $B$. This means that two-hop attention in MAGNA is context (node $D$ ) dependent.

Oono and Suzuki, 2020] developed attention mechanism for Graph Neural Networks (GNNs), which compute attention scores between nodes connected by an edge, allowing the model to attend to messages of node's neighbors.

However, such attention computation on pairs of nodes connected by edges implies that a node can only attend to its immediate neighbors to compute its (next layer) representation. This implies that receptive field of a single GNN layer is restricted to one-hop network neighborhoods. Although stacking multiple GAT layers could in principle enlarge the receptive field and learn non-neighboring interactions, such deep GAT architectures suffer from the oversmoothing problem [Wang et al., 2019a; Liu et al., 2019; Oono and Suzuki, 2020] and do not perform well. Furthermore, edge attention in a single GAT layer is based solely on representations of the two nodes at the edge endpoints, and does not depend on their graph neighborhood context. In other words, the one-hop attention mechanism in GATs limits their ability to explore the relationship between the broader graph structure. While previous works [Xu et al., 2018; Klicpera et al., 2019b] have shown advantages in performing multi-hop message-passing in a single layer, these ap- 
proaches are not graph-attention based. Therefore, incorporating multi-hop neighboring context into the attention computation in graph neural networks remains to be explored.

Here we present Multi-hop Attention Graph Neural Network (MAGNA), an effective multi-hop self-attention mechanism for graph structured data. MAGNA uses a novel graph attention diffusion layer (Figure 1), where we first compute attention weights on edges (represented by solid arrows), and then compute self-attention weights (dotted arrows) between disconnected pairs of nodes through an attention diffusion process using the attention weights on the edges.

Our model has two main advantages: (1) MAGNA captures long-range interactions between nodes that are not directly connected but may be multiple hops away. Thus the model enables effective long-range message passing, from important nodes multiple hops away. (2) The attention computation in MAGNA is context-dependent. The attention value in GATs [Veličković et al., 2018] only depends on node representations of the previous layer, and is zero between nonconnected pairs of nodes. In contrast, for any pair of nodes within a chosen multi-hop neighborhood, MAGNA computes attention by aggregating the attention scores over all the possible paths (length $\geq 1$ ) connecting the two nodes.

Mathematically we show that MAGNA places a Personalized Page Rank (PPR) prior on the attention values. We further apply spectral graph analysis to show that MAGNA emphasizes on large-scale graph structure and lowering highfrequency noise in graphs. Specifically, MAGNA enlarges the lower Laplacian eigen-values, which correspond to the large-scale structure in the graph, and suppresses the higher Laplacian eigen-values which correspond to more noisy and fine-grained information in the graph.

We experiment on standard datasets for semi-supervised node classification as well as knowledge graph completion. Experiments show that MAGNA achieves state-of-the-art results: MAGNA achieves up to $5.7 \%$ relative error reduction over previous state-of-the-art on Cora, Citeseer, and Pubmed. MAGNA also obtains better performance on a large-scale Open Graph Benchmark dataset. On knowledge graph completion, MAGNA advances state-of-the-art on WN18RR and FB15k-237 across four metrics, with the largest gain of $7.1 \%$ in the metric of Hit at 1 .

Furthermore, we show that MAGNA with just 3 layers and 6 hop wide attention per layer significantly out-performs GAT with 18 layers, even though both architectures have the same receptive field. Moreover, our ablation study reveals the synergistic effect of the essential components of MAGNA, including layer normalization and multi-hop diffused attention. We further observe that compared to GAT, the attention values learned by MAGNA have higher diversity, indicating the ability to better pay attention to important nodes.

\section{Multi-hop Attention Graph Neural Network (MAGNA)}

We first discuss the background and explain the novel multihop attention diffusion module and the MAGNA architecture.

\subsection{Preliminaries}

Let $\mathcal{G}=(\mathcal{V}, \mathcal{E})$ be a given graph, where $\mathcal{V}$ is the set of $N_{n}$ nodes, $\mathcal{E} \subseteq \mathcal{V} \times \mathcal{V}$ is the set of $N_{e}$ edges connecting $M$ pairs of nodes in $\overline{\mathcal{V}}$. Each node $v \in \mathcal{V}$ and each edge $e \in \mathcal{E}$ are associated with their type mapping functions: $\phi: \mathcal{V} \rightarrow \mathcal{T}$ and $\psi:$ $\mathcal{E} \rightarrow \mathcal{R}$. Here $\mathcal{T}$ and $\mathcal{R}$ denote the sets of node types (labels) and edge/relation types. Our framework supports learning on heterogeneous graphs with multiple elements in $\mathcal{R}$.

A general Graph Neural Network (GNN) approach learns an embedding that maps nodes and/or edge types into a continuous vector space. Let $\boldsymbol{X} \in \mathbb{R}^{N_{n} \times d_{n}}$ and $\boldsymbol{R} \in \mathbb{R}^{N_{r} \times d_{r}}$ be the node embedding and edge/relation type embedding, where $N_{n}=|\mathcal{V}|, N_{r}=|\mathcal{R}|, d_{n}$ and $d_{r}$ represent the embedding dimension of node and edge/relation types, each row $\boldsymbol{x}_{i}=\boldsymbol{X}[i:]$ represents the embedding of node $v_{i}(1 \leq i \leq$ $\left.N_{n}\right)$, and $\boldsymbol{r}_{j}=\boldsymbol{R}[j:]$ represents the embedding of relation $r_{j}\left(1 \leq j \leq N_{r}\right)$. MAGNA builds on GNNs, while bringing together the benefits of Graph Attention and Diffusion techniques.

\subsection{Multi-hop Attention Diffusion}

We first introduce attention diffusion to compute the multihop attention directly, which operates on the MAGNA's attention scores at each layer. The input to the attention diffusion operator is a set of triples $\left(v_{i}, r_{k}, v_{j}\right)$, where $v_{i}, v_{j}$ are nodes and $r_{k}$ is the edge type. MAGNA first computes the attention scores on all edges. The attention diffusion module then computes the attention values between pairs of nodes that are not directly connected by an edge, based on the edge attention scores, via a diffusion process. The attention diffusion module can then be used as a component in MAGNA architecture, which we will further elaborate in Section 2.3.

Edge Attention Computation At each layer $l$, a vector message is computed for each triple $\left(v_{i}, r_{k}, v_{j}\right)$. To compute the representation of $v_{j}$ at layer $l+1$, all messages from triples incident to $v_{j}$ are aggregated into a single message, which is then used to update $v_{j}^{l+1}$.

In the first stage, the attention score $s$ of an edge $\left(v_{i}, r_{k}, v_{j}\right)$ is computed by the following:

$$
s_{i, k, j}^{(l)}=\delta\left(\boldsymbol{v}_{a}^{(l)} \tanh \left(\boldsymbol{W}_{h}^{(l)} \boldsymbol{h}_{\boldsymbol{i}}^{(l)}\left\|\boldsymbol{W}_{t}^{(l)} \boldsymbol{h}_{\boldsymbol{j}}^{(\boldsymbol{l})}\right\| \boldsymbol{W}_{r}^{(l)} \boldsymbol{r}_{k}\right)\right)
$$

where $\delta=$ LeakyReLU, $\boldsymbol{W}_{h}^{(l)}, \boldsymbol{W}_{t}^{(l)} \in \mathbb{R}^{d^{(l)} \times d^{(l)}}, \boldsymbol{W}_{r}^{(l)} \in$ $\mathbb{R}^{d^{(l)} \times d_{r}}$ and $\boldsymbol{v}_{a}^{(l)} \in \mathbb{R}^{1 \times 3 d^{(l)}}$ are the trainable weights shared by $l$-th layer. $\boldsymbol{h}_{i}^{(l)} \in \mathbb{R}^{d^{(l)}}$ represents the embedding of node $i$ at $l$-th layer, and $\boldsymbol{h}_{i}^{(0)}=\boldsymbol{x}_{i} . \boldsymbol{r}_{k}$ is the trainable relation embedding of the $k$-th relation type $r_{k}\left(1 \leq k \leq N_{r}\right)$, and $a \| b$ denotes concatenation of embedding vectors $a$ and $b$. For graphs with no relation type, we treat as a degenerate categorical distribution with 1 category ${ }^{1}$.

Applying Eq. 1 on each edge of the graph $\mathcal{G}$, we obtain an attention score matrix $\boldsymbol{S}^{(l)}$ :

$$
\boldsymbol{S}_{i, j}^{(l)}= \begin{cases}s_{i, k, j}^{(l)}, & \text { if }\left(v_{i}, r_{k}, v_{j}\right) \text { appears in } \mathcal{G} \\ -\infty, & \text { otherwise }\end{cases}
$$

\footnotetext{
${ }^{1}$ In this case, we can view that there is only one "pseudo" relation type (category), i.e., $N_{r}=1$
} 
Subsequently we obtain the attention matrix $\boldsymbol{A}^{(l)}$ by performing row-wised softmax over the score matrix $\boldsymbol{S}^{(l)}: \boldsymbol{A}^{(l)}=$ $\operatorname{softmax}\left(\boldsymbol{S}^{(l)}\right) . \quad \boldsymbol{A}_{i j}^{(l)}$ denotes the attention value at layer $l$ when aggregating message from node $j$ to node $i$.

Attention Diffusion for Multi-hop Neighbors In the second stage, we further enable attention between nodes that are not directly connected in the network. We achieve this via the following attention diffusion procedure. The procedure computes the attention scores of multi-hop neighbors via graph diffusion based on the powers of the 1-hop attention matrix A:

$$
\mathcal{A}=\sum_{i=0}^{\infty} \theta_{i} \boldsymbol{A}^{i} \text { where } \sum_{i=0}^{\infty} \theta_{i}=1 \text { and } \theta_{i}>0
$$

where $\theta_{i}$ is the attention decay factor and $\theta_{i}>\theta_{i+1}$. The powers of attention matrix, $A^{i}$, give us the number of relation paths from node $h$ to node $t$ of length up to $i$, increasing the receptive field of the attention (Figure 1). Importantly, the mechanism allows the attention between two nodes to not only depend on their previous layer representations, but also taking into account of the paths between the nodes, effectively creating attention shortcuts between nodes that are not directly connected (Figure 1). Attention through each path is also weighted differently, depending on $\theta$ and the path length.

In our implementation we utilize the geometric distribution $\theta_{i}=\alpha(1-\alpha)^{i}$, where $\alpha \in(0,1]$. The choice is based on the inductive bias that nodes further away should be weighted less in message aggregation, and nodes with different relation path lengths to the target node are sequentially weighted in an independent manner. In addition, notice that if we define $\theta_{0}$ $=\alpha \in(0,1], \boldsymbol{A}^{0}=\boldsymbol{I}$, then Eq. 3 gives the Personalized Page Rank (PPR) procedure on the graph with the attention matrix $\boldsymbol{A}$ and teleport probability $\alpha$. Hence the diffused attention weights, $\mathcal{A}_{i j}$, can be seen as the influence of node $j$ to node $i$. We further elaborate the significance of this observation in Section 4.3.

We can also view $\mathcal{A}_{i j}$ as the attention value of node $j$ to $i$ since $\sum_{j=1}^{N_{n}} \mathcal{A}_{i j}=1 .^{2}$ We then define the graph attention diffusion based feature aggregation as

$$
\operatorname{AttDiff}\left(\mathcal{G}, \boldsymbol{H}^{(l)}, \Theta\right)=\mathcal{A} \boldsymbol{H}^{(l)},
$$

where $\Theta$ is the set of parameters for computing attention. Thanks to the diffusion process defined in Eq. 3, MAGNA uses the same number of parameters as if we were only computing attention between nodes connected via edges. This ensures runtime efficiency (refer to Appendix ${ }^{3}$ A for complexity analysis) and model generalization.

Approximate Computation for Attention Diffusion For large graphs computing the exact attention diffusion matrix $\mathcal{A}$ using Eq. 3 may be prohibitively expensive, due to computing the powers of the attention matrix [Klicpera et al., 2019a]. To resolve this bottleneck, we proceed as follows: Let $\boldsymbol{H}^{(l)}$ be the input entity embedding of the $l$-th layer $\left(\boldsymbol{H}^{(0)}=\boldsymbol{X}\right)$

\footnotetext{
${ }^{2}$ Obtained by the definition $\boldsymbol{A}^{(l)}=\operatorname{softmax}\left(\boldsymbol{S}^{(l)}\right)$ and Eq. 3 .

${ }^{3}$ Appendix can be found via https://arxiv.org/abs/2009.14332
}

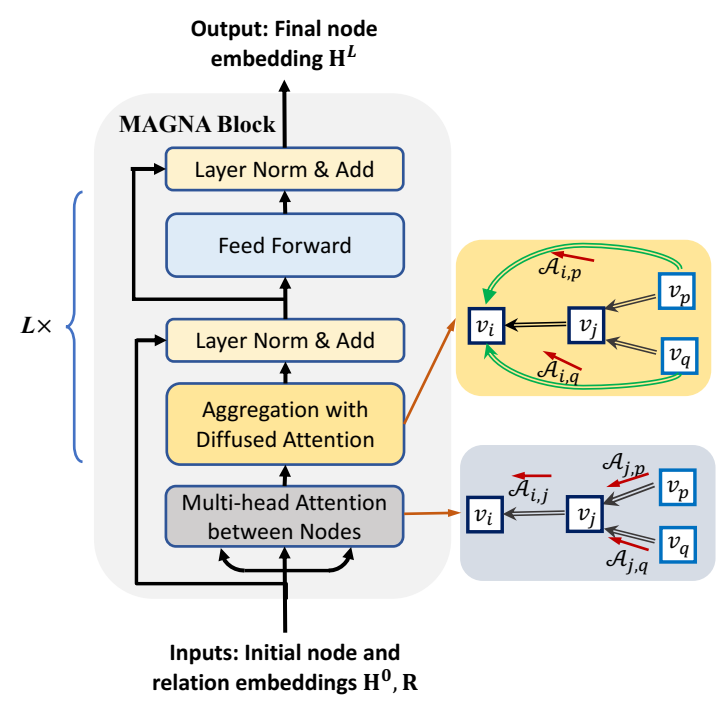

Figure 2: MAGNA Architecture. Each MAGNA block consists of attention computation, attention diffusion, layer normalization, feed forward layers, and 2 residual connections for each block. MAGNA blocks can be stacked to constitute a deep model. As illustrated on the right, context-dependent attention is achieved via the attention diffusion process. Here $v_{i}, v_{j}, v_{p}, v_{q} \in \mathcal{V}$ are nodes in the graph.

and $\theta_{i}=\alpha(1-\alpha)^{i}$. Since MAGNA only requires aggregation via $\mathcal{A} \boldsymbol{H}^{(l)}$, we can approximate $\mathcal{A} \boldsymbol{H}^{(l)}$ by defining a sequence $Z^{(K)}$ which converges to the true value of $\mathcal{A} \boldsymbol{H}^{(l)}$ (Proposition 1) as $K \rightarrow \infty$ :

$$
\boldsymbol{Z}^{(0)}=\boldsymbol{H}^{(l)}, \boldsymbol{Z}^{(k+1)}=(1-\alpha) \boldsymbol{A} \boldsymbol{Z}^{(k)}+\alpha \boldsymbol{Z}^{(0)},
$$

where $0 \leq k<K$.

Proposition 1. $\lim _{K \rightarrow \infty} Z^{(K)}=\mathcal{A} \boldsymbol{H}^{(l)}$

In the Appendix we give the proof which relies on the expansion of Eq. 5 .

Using the above approximation, the complexity of attention computation with diffusion is still $O(|E|)$, with a constant factor corresponding to the number of hops $K$. In practice, we find that choosing the values of $K$ such that $3 \leq K \leq 10$ results in good model performance. Many real-world graphs exhibit small-world property, in which case even a smaller value of $K$ is sufficient. For graphs with larger diameter, we choose larger $K$, and lower the value of $\alpha$.

\subsection{Multi-hop Attention based GNN Architecture}

Figure 2 provides an architecture overview of the MAGNA Block that can be stacked multiple times.

Multi-head Graph Attention Diffusion Layer Multi-head attention [Vaswani et al., 2017; Veličković et al., 2018] is used to allow the model to jointly attend to information from different representation sub-spaces at different viewpoints. In Eq. 6, the attention diffusion for each head $i$ is computed separately with Eq. 4, and aggregated:

$$
\begin{aligned}
\hat{\boldsymbol{H}}^{(l)} & =\operatorname{MultiHead}\left(\mathcal{G}, \tilde{\boldsymbol{H}}^{(l)}\right)=\left(\|_{i=1}^{M} \operatorname{head}_{i}\right) \boldsymbol{W}_{o} \\
\operatorname{head}_{i} & =\operatorname{AttDiff}\left(\mathcal{G}, \tilde{\boldsymbol{H}}^{(l)}, \Theta_{i}\right), \tilde{\boldsymbol{H}}^{(l)}=\operatorname{LN}\left(\boldsymbol{H}^{(l)}\right),
\end{aligned}
$$


where $\|$ denotes concatenation and $\Theta_{i}$ are the parameters in Eq. 1 for the $i$-th head $(1 \leq i \leq M), \boldsymbol{W}_{o}$ represents a parameter matrix, and LN = LayerNorm. Since we calculate the attention diffusion in a recursive way in Eq. 5, we add layer normalization which helpful to stabilize the recurrent computation procedure [Ba et al., 2016].

Deep Aggregation Moreover our MAGNA block contains a fully connected feed-forward sub-layer, which consists of a two-layer feed-forward network. We also add the layer normalization and residual connection in both sub-layers, allowing for a more expressive aggregation step for each block [Xiong et al., 2020]:

$$
\begin{aligned}
& \hat{\boldsymbol{H}}^{(l+1)}=\hat{\boldsymbol{H}}^{(l)}+\boldsymbol{H}^{(l)} \\
& \boldsymbol{H}^{(l+1)}=\boldsymbol{W}_{2}^{(l)} \operatorname{ReLU}\left(\boldsymbol{W}_{1}^{(l)} \operatorname{LN}\left(\hat{\boldsymbol{H}}^{(l+1)}\right)\right)+\hat{\boldsymbol{H}}^{(l+1)}
\end{aligned}
$$

MAGNA generalizes GAT MAGNA extends GAT via the diffusion process. The feature aggregation in GAT is $\boldsymbol{H}^{(l+1)}=\sigma\left(\boldsymbol{A} \boldsymbol{H}^{(l)} \boldsymbol{W}^{(l)}\right)$, where $\sigma$ represents the activation function. We can divide GAT layer into two components as follows:

$$
\boldsymbol{H}^{(l+1)}=\underbrace{\sigma}_{(2)}(\underbrace{\boldsymbol{A} \boldsymbol{H}^{(l)} \boldsymbol{W}^{(l)}}_{(1)}) .
$$

In component (1), MAGNA removes the restriction of attending to direct neighbors, without requiring additional parameters as $\mathcal{A}$ is induced from $\boldsymbol{A}$. For component (2) MAGNA uses layer normalization and deep aggregation to achieve higher expressive power compared to elu nonlinearity in GAT.

\section{Analysis of Graph Attention Diffusion}

In this section, we investigate the benefits of MAGNA from the viewpoint of discrete signal processing on graphs [Sandryhaila and Moura, 2013]. Our first result demonstrates that MAGNA can better capture large-scale structural information. Our second result explores the relation between MAGNA and Personalized PageRank (PPR).

\subsection{Spectral Properties of Graph Attention Diffusion}

We view the attention matrix $\boldsymbol{A}$ of GAT, and $\mathcal{A}$ of MAGNA as weighted adjacency matrices, and apply Graph Fourier transform and spectral analysis (details in Appendix) to show the effect of MAGNA as a graph low-pass filter, being able to more effectively capture large-scale structure in graphs. By Eq. 3, the sum of each row of either $\mathcal{A}$ or $\boldsymbol{A}$ is 1 . Hence the normalized graph Laplacians are $\hat{\boldsymbol{L}}_{\text {sym }}=\boldsymbol{I}-\mathcal{A}$ and $\boldsymbol{L}_{\text {sym }}=\boldsymbol{I}-\boldsymbol{A}$ for $\mathcal{A}$ and $\boldsymbol{A}$ respectively. We can get the following proposition:

Proposition 2. Let $\hat{\lambda}_{i}^{g}$ and $\lambda_{i}^{g}$ be the $i$-th eigeinvalues of $\hat{\boldsymbol{L}}_{\text {sym }}$ and $\boldsymbol{L}_{\text {sym }}$.

$$
\frac{\hat{\lambda}_{i}^{g}}{\lambda_{i}^{g}}=\frac{1-\frac{\alpha}{1-(1-\alpha)\left(1-\lambda_{i}^{g}\right)}}{\lambda_{i}^{g}}=\frac{1}{\frac{\alpha}{1-\alpha}+\lambda_{i}^{g}} .
$$

Refer to Appendix for the proof. We additionally have $\lambda_{i}^{g} \in[0,2]$ (proved by [Ng et al., 2002]). Eq. 9 shows that when $\lambda_{i}^{g}$ is small such that $\frac{\alpha}{1-\alpha}+\lambda_{i}^{g}<1$, then $\hat{\lambda}_{i}^{g}>\lambda_{i}^{g}$, whereas for large $\lambda_{i}^{g}, \hat{\lambda}_{i}^{g}<\lambda_{i}^{g}$. This relation indicates that the use of $\mathcal{A}$ increases smaller eigenvalues and decreases larger eigenvalues ${ }^{4}$. See Section 4.3 for its empirical evidence. The low-pass effect increases with smaller $\alpha$.

The eigenvalues of the low-frequency signals describe the large-scale structure in the graph [ $\mathrm{Ng}$ et al., 2002] and have been shown to be crucial in graph tasks [Klicpera et al., 2019b]. As $\lambda_{i}^{g} \in[0,2]\left[\mathrm{Ng}\right.$ et al., 2002] and $\frac{\alpha}{1-\alpha}>0$, the reciprocal format in Eq. 9 will amplify the ratio of lower eigenvalues to the sum of all eigenvalues. In contrast, high eigenvalues corresponding to noise are suppressed.

\subsection{Personalized PageRank Meets Graph Attention Diffusion}

We can also view the attention matrix $\boldsymbol{A}$ as a random walk matrix on graph $\mathcal{G}$ since $\sum_{j=1}^{N_{n}} \boldsymbol{A}_{i, j}=1$ and $\boldsymbol{A}_{i, j}>0$. If we perform Personalized PageRank (PPR) with parameter $\alpha \in$ $(0,1]$ on $\mathcal{G}$ with transition matrix $\boldsymbol{A}$, the fully Personalized PageRank [Lofgren, 2015] is defined as:

$$
\boldsymbol{A}_{p p r}=\alpha(\boldsymbol{I}-(1-\alpha) \boldsymbol{A})^{-1}
$$

Using the power series expansion for the matrix inverse, we obtain

$$
\boldsymbol{A}_{p p r}=\alpha \sum_{i=0}^{\infty}(1-\alpha)^{i} \boldsymbol{A}^{i}=\sum_{i=0}^{\infty} \alpha(1-\alpha)^{i} \boldsymbol{A}^{i}
$$

Comparing to the diffusion Equation 3 with $\theta_{i}=\alpha(1-\alpha)^{i}$, we have the following proposition.

Proposition 3. Graph attention diffusion defines a personalized page rank with parameter $\alpha \in(0,1]$ on $\mathcal{G}$ with transition matrix $\boldsymbol{A}$, i.e., $\mathcal{A}=\boldsymbol{A}_{\text {ppr }}$.

The parameter $\alpha$ in MAGNA is equivalent to the teleport probability of PPR. PPR provides a good relevance score between nodes in a weighted graph (the weights from the attention matrix $\boldsymbol{A}$ ). In summary, MAGNA places a PPR prior over node pairwise attention scores: the diffused attention between node $i$ and $j$ depends on the attention scores on the edges of all paths between $i$ and $j$.

\section{Experiments}

We evaluate MAGNA on two classical tasks ${ }^{5}$ : (1) on node classification we achieve an average of $5.7 \%$ relative error reduction; (2) on knowledge graph completion we achieve $7.1 \%$ relative improvement in the Hit@ 1 metric. ${ }^{6}$

\subsection{Task 1: Node Classification}

Datasets We employ four benchmark datasets for node classification: (1) standard citation network benchmarks Cora, Citeseer and Pubmed [Sen et al., 2008; Kipf and Welling, 2016]; and (2) a benchmark dataset ogbn-arxiv on

\footnotetext{
${ }^{4}$ The eigenvalues of $\mathcal{A}$ and $\boldsymbol{A}$ correspond to the same eigenvectors, as shown in Proposition 2 in Appendix.

${ }^{5}$ All datasets are public; code will be released after publication.

${ }^{6}$ Please see the definitions of these two tasks in Appendix.
} 


\begin{tabular}{|c|c|c|c|c|}
\hline \multirow{10}{*}{ 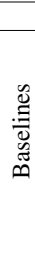 } & Models & Cora & Citeseer & Pubmed \\
\hline & GCN \Kipf and Welling, 2016] & 81.5 & 70.3 & 79.0 \\
\hline & Cheby [Defferrard et al., 2016] & 81.2 & 69.8 & 74.4 \\
\hline & DualGCN [Zhuang and Ma, 2018] & 83.5 & 72.6 & 80.0 \\
\hline & JKNet $\left[\right.$ Xu et al., 2018] ${ }^{\star}$ & 81.1 & 69.8 & 78.1 \\
\hline & LGCN [Gao et al., 2018] & $83.3 \pm 0.5$ & $73.0 \pm 0.6$ & $79.5 \pm 0.2$ \\
\hline & Diff-GCN [Klicpera et al., 2019b] & $83.6 \pm 0.2$ & $73.4 \pm 0.3$ & $79.6 \pm 0.4$ \\
\hline & APPNP [Klicpera et al., 2019a] & $84.3 \pm 0.2$ & $71.1 \pm 0.4$ & $79.7 \pm 0.3$ \\
\hline & g-U-Nets [Gao and Ji, 2019] & $84.4 \pm 0.6$ & $73.2 \pm 0.5$ & $79.6 \pm 0.2$ \\
\hline & GAT [Veličković et al., 2018] & $83.0 \pm 0.7$ & $72.5 \pm 0.7$ & $79.0 \pm 0.3$ \\
\hline \multirow{5}{*}{$\frac{2}{4}$} & No LayerNorm & $83.8 \pm 0.6$ & $71.1 \pm 0.5$ & $79.8 \pm 0.2$ \\
\hline & No Diffusion & $83.0 \pm 0.4$ & $71.6 \pm 0.4$ & $79.3 \pm 0.3$ \\
\hline & No Feed-Forward ${ }^{\diamond}$ & $84.9 \pm 0.4$ & $72.2 \pm 0.3$ & $80.9 \pm 0.3$ \\
\hline & No (LayerNorm + Feed-Forward) & $84.3 \pm 0.6$ & $72.6 \pm 0.4$ & $79.6 \pm 0.4$ \\
\hline & MAGNA & $\mathbf{8 5 . 4} \pm 0.6$ & $73.7 \pm 0.5$ & $81.4 \pm 0.2$ \\
\hline
\end{tabular}

Table 1: Node classification accuracy on Cora, Citeseer, Pubmed. MAGNA achieves state-of-the-art.

$170 \mathrm{k}$ nodes and $1.2 \mathrm{~m}$ edges from the Open Graph Benchmark [Weihua $\mathrm{Hu}, 2020$ ]. We follow the standard data splits for all datasets. Further information about these datasets is summarized in the Appendix.

Baselines We compare against a comprehensive suite of state-of-the-art GNN methods including: GCNs [Kipf and Welling, 2016], Chebyshev filter based GCNs [Defferrard et al., 2016], DualGCN [Zhuang and Ma, 2018], JKNet [Xu et al., 2018], LGCN [Gao et al., 2018], Diffusion-GCN (Diff-GCN) [Klicpera et al., 2019b], APPNP [Klicpera et al., 2019a], Graph U-Nets (g-U-Nets) [Gao and Ji, 2019], and GAT [Veličković et al., 2018].

Experimental Setup For datasets Cora, Citeseer and Pubmed, we use 6 MAGNA blocks with hidden dimension 512 and 8 attention heads. For the large-scale ogbn-arxiv dataset, we use 2 MAGNA blocks with hidden dimension 128 and 8 attention heads. Refer to Appendix for detailed description of all hyper-parameters and evaluation settings.

Results MAGNA achieves the best on all datasets (Tables 1 and 2) ${ }^{7}$, out-performing mutlihop baselines such as Diffusion GCN, APPNP and JKNet. The baseline performance and their embedding dimensions are from the previous papers. Appendix Table 6 further demonstrates that large 512 dimension embedding only benefits the expressive MAGNA, whereas GAT and Diffusion GCN performance degrades.

Ablation study We report (Table 1) the model performance after removing each component of MAGNA (layer normalization, attention diffusion and feed forward layers) from every MAGNA layer. Note that the model is equivalent to GAT without these three components. We observe that diffusion and layer normalization play a crucial role in improving the node classification performance for all datasets. Since MAGNA computes attention diffusion in a recursive manner, layer normalization is crucial in ensuring training stability [Ba et al., 2016]. Meanwhile, comparing to GAT (see the next-to-last row of Table 1), attention diffusion allows multihop attention in every layer to benefit node classification.

\footnotetext{
${ }^{7}$ We also compared to GAT and Diffusion-GCN (with LayerNorm and feed-forward Layer) over random splits in Appendix.
}

\subsection{Task 2: Knowledge Graph Completion}

Datasets We evaluate MAGNA on standard benchmark knowledge graphs: WN18RR [Dettmers et al., 2018] and FB15K-237 [Toutanova and Chen, 2015]. See the statistics of these KGs in Appendix.

Baselines We compare MAGNA with state-of-the-art baselines, including (1) translational distance based models: TransE [Bordes et al., 2013] and its latest extension RotatE [Sun et al., 2019], OTE [Tang et al., 2020] and ROTH [Chami et al., 2020]; (2) semantic matching based models: ComplEx [Trouillon et al., 2016], QuatE [Zhang et al., 2019], CoKE [Wang et al., 2019b], ConvE [Dettmers et al., 2018], DistMult [Yang et al., 2015], TuckER [Balazevic et al., 2019] and AutoSF [Zhang et al., 2020]; (3) GNN-based models: R-GCN [Schlichtkrull et al., 2018], SACN [Shang et al., 2019] and A2N [Bansal et al., 2019].

Experimental Setup We use the multi-layer MAGNA as encoder for both FB15k-237 and WN18RR. We randomly initialize the entity embedding and relation embedding as the input of the encoders, and set the dimensionality of the initialized entity/relation vector as 100 used in DistMult [Yang et al., 2015]. We select other MAGNA model hype-parameters, including number of layers, hidden dimension, head number, top- $k$, learning rate, hop number, teleport probability $\alpha$ and dropout ratios (see the settings of these parameter in Appendix), by a random search during the training.

Training procedure We use the standard training procedure used in previous $\mathrm{KG}$ embedding models [Balazevic et al., 2019; Dettmers et al., 2018] (Appendix for details). We follow an encoder-decoder framework: The encoder applies the proposed MAGNA model to compute the entity embeddings. The decoder makes link prediction given the embeddings. To show the power of MAGNA, we employ a simple decoder DistMult [Yang et al., 2015].

Evaluation We use the standard split for the benchmarks, and the standard testing procedure of predicting tail (head) entity given the head (tail) entity and relation type. We exactly follow the evaluation used by all previous works, namely the Mean Reciprocal Rank (MRR), Mean Rank (MR), and hit rate at $K(\mathrm{H} @ \mathrm{~K})$. See Appendix for a detailed description of this standard setup.

Results MAGNA achieves new state-of-the-art in knowledge graph completion on all four metrics (Table 3). MAGNA compares favourably to both the most recent shallow embedding methods (QuatE), and deep embedding methods (SACN). Note that with the same decoder (DistMult), MAGNA using its own embeddings achieves drastic improvements over using the corresponding DistMult embeddings.

\subsection{MAGNA Model Analysis}

Here we present (1) spectral analysis results, (2) robustness to hyper-parameter changes, and (3) attention distribution analysis to show the strengths of MAGNA.

Spectral Analysis: Why MAGNA works for node classification? We compute the eigenvalues of the graph Laplacian 


\begin{tabular}{lcccccc}
\hline & GCN & GraphSAGE & JKNet & DAGNN & GaAN & \\
Data & [Kipf and Welling, 2016] & [Hamilton et al., 2017] & [Xu et al., 2018] & [Liu et al., 2020] & [Zhang et al., 2018] & MAGNA \\
\hline ogbn-arxiv & $71.74 \pm 0.29$ & $71.49 \pm 0.27$ & $72.19 \pm 0.21$ & $72.09 \pm 0.25$ & $71.97 \pm 0.24$ & $\mathbf{7 2 . 7 6} \pm 0.14$ \\
\hline
\end{tabular}

Table 2: Node classification accuracy on the OGB Arxiv dataset.

\begin{tabular}{|c|c|c|c|c|c|c|c|c|c|c|}
\hline \multirow{2}{*}{ Models } & \multicolumn{5}{|c|}{ WN18RR } & \multicolumn{5}{|c|}{ FB15k-237 } \\
\hline & MR & MRR & H@1 & $\mathrm{H} @ 3$ & $\mathrm{H} @ 10$ & MR & MRR & $\mathrm{H} @ 1$ & $\mathrm{H} @ 3$ & H@10 \\
\hline TransE [Bordes et al., 2013] & 3384 & .226 & - & - & .501 & 357 & .294 & - & - & .465 \\
\hline RotatE [Sun et al., 2019] & 3340 & .476 & .428 & .492 & .571 & 177 & .338 & .241 & .375 & .533 \\
\hline OTE [Tang et al., 2020] & - & .491 & .442 & .511 & .583 & - & .361 & .267 & .396 & .550 \\
\hline ROTH [Chami et al., 2020] & - & .496 & .449 & .514 & .586 & - & .344 & .246 & .380 & .535 \\
\hline ComplEx [Trouillon et al., 2016] & 5261 & .44 & .41 & .46 & .51 & 339 & .247 & .158 & .275 & .428 \\
\hline QuatE [Zhang et al., 2019] & 2314 & .488 & .438 & .508 & .582 & - & .366 & .271 & .401 & .556 \\
\hline CoKE [Wang et al., 2019b] & - & .475 & .437 & .490 & .552 & - & .361 & .269 & .398 & .547 \\
\hline ConvE [Dettmers et al., 2018] & 4187 & .43 & .40 & .44 & .52 & 244 & .325 & .237 & .356 & .501 \\
\hline DistMult [Yang et al., 2015] & 5110 & .43 & .39 & .44 & .49 & 254 & .241 & .155 & .263 & .419 \\
\hline TuckER [Balazevic et al., 2019] & - & .470 & .443 & .482 & .526 & - & .358 & .266 & .392 & .544 \\
\hline AutoSF [Zhang et al., 2020] & - & .490 & .451 & - & .567 & - & .360 & .267 & - & .552 \\
\hline R-GCN [Schlichtkrull et al., 2018] & - & - & - & - & - & - & .249 & .151 & .264 & .417 \\
\hline SACN [Shang et al., 2019] & - & .47 & .43 & .48 & .54 & - & .35 & .26 & .39 & .54 \\
\hline A2N [Bansal et al., 2019] & - & .45 & .42 & .46 & .51 & - & .317 & .232 & .348 & .486 \\
\hline MAGNA + DistMult & 2545 & .502 & .459 & .519 & .589 & 138 & .369 & .275 & .409 & .563 \\
\hline
\end{tabular}

Table 3: KG Completion on WN18RR and FB15k-237. MAGNA achieves state of the art.
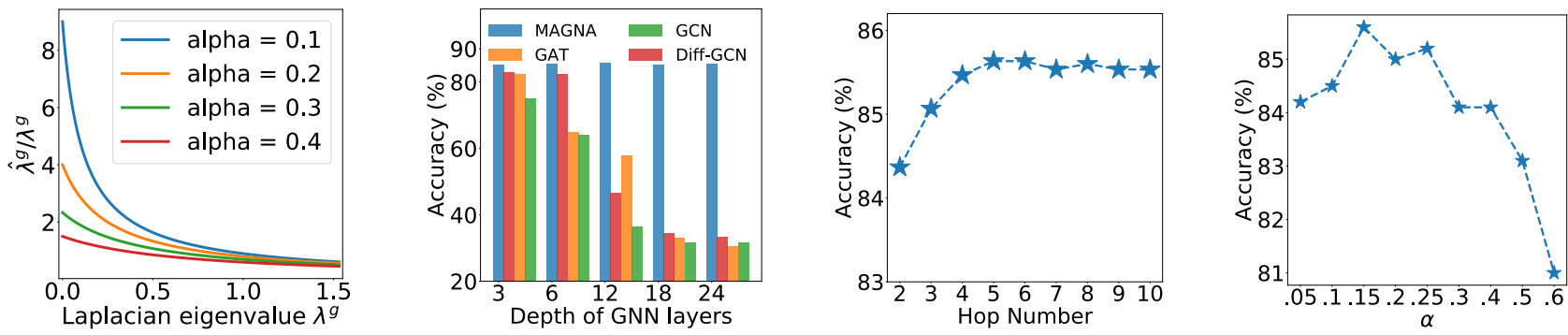

Figure 3: Analysis of MAGNA on Cora. (a) Influence of MAGNA on Laplacian eigenvalues. (b) Effect of depth on performance. (c) Effect of hop number $K$ on performance. (d) Effect of teleport probability $\alpha$.

of the attention matrix $\mathbf{A}, \hat{\lambda}_{i}^{g}$, and compare to that of the diffused matrix $\mathcal{A}, \lambda_{i}^{g}$. Figure 3 (a) shows the ratio $\hat{\lambda}_{i}^{g} / \lambda_{i}^{g}$ on the Cora dataset. Low eigenvalues corresponding to large-scale structure in the graph are amplified (up to a factor of 8), while high eigenvalues corresponding to eigenvectors with noisy information are suppressed [Klicpera et al., 2019b].

MAGNA Model Depth Here we conduct experiments by varying the number of GCN, Diffusion-GCN (PPR based) GAT and our MAGNA layers to be 3, 6, 12, 18 and 24 for node classification on Cora. Results in Fig. 3 (b) show that deep GCN, Diffusion-GCN and GAT (even with residual connection) suffer from degrading performance, due to the over-smoothing problem [Li et al., 2018; Wang et al., 2019a]. In contrast, the MAGNA model achieves consistent best results even with 18 layers, making deep MAGNA model robust and expressive. Notice that GAT with 18 layers cannot out-perform MAGNA with 3 layers and $K=6$ hops, although they have the same receptive field.

Effect of $K$ and $\alpha \quad$ Figs. 3 (c) and (d) report the effect of hop number $K$ and teleport probability $\alpha$ on model performance. We observe significant increase in performance when considering multi-hop neighbors information $(K>1)$. However, increasing the hop number $K$ has a diminishing returns, for $K \geq 6$. Moreover, we find that the optimal $K$ is correlated with the largest node average shortest path distance (e.g., 5.27 for Cora). This provides a guideline for choosing the best $K$.

We also observe that the accuracy drops significantly for larger $\alpha>0.25$. This is because small $\alpha$ increases the lowpass effect (Fig. 3 (a)). However, $\alpha$ being too small causes the model to only focus on the most large-scale graph structure and have lower performance.

Attention Distribution Last we also analyze the learned attention scores of GAT and MAGNA. We first define a discrepancy metric over the attention matrix $\boldsymbol{A}$ for node $v_{i}$ as $\Delta_{i}=\frac{\left\|\boldsymbol{A}_{[i, j}-U_{i}\right\|}{\operatorname{degree}\left(v_{i}\right)}$ [Shanthamallu et al., 2020], where $U_{i}$ is the uniform distribution score for the node $v_{i} . \Delta_{i}$ gives a measure of how much the learnt attention deviates from an uninformative uniform distribution. Large $\Delta_{i}$ indicates more meaningful attention scores. Fig. 4 shows the distribution of the discrepancy metric of the attention matrix of the 1st head w.r.t. the first layer of MAGNA and GAT. Observe that at- 


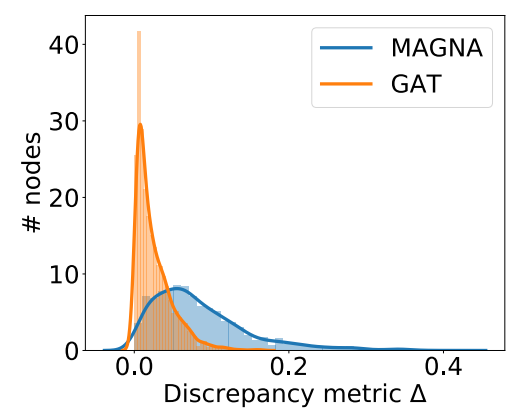

Figure 4: Attention weight distribution on Cora.

tention scores learned in MAGNA have much larger discrepancy. This shows that MAGNA is more powerful than GAT in distinguishing important nodes and assigns attention scores accordingly.

\section{Related Work}

Our proposed MAGNA belongs to the family of Graph Neural Network (GNN) models [Battaglia et al., 2018; Wu et al., 2020; Kipf and Welling, 2016; Hamilton et al., 2017], while taking advantage of graph attention and diffusion techniques.

Graph Attention Neural Networks (GATs) generalize attention operation to graph data. GATs allow for assigning different importance to nodes of the same neighborhood at the feature aggregation step [Veličković et al., 2018]. Based on such framework, different attention-based GNNs have been proposed, including GaAN [Zhang et al., 2018], AGNN [Thekumparampil et al., 2018], GeniePath [Liu et al., 2019]. However, these models only consider direct neighbors for each layer of feature aggregation, and suffer from oversmoothing when they go deep [Wang et al., 2019a].

Diffusion based Graph Neural Network Recently Graph Diffusion Convolution [Klicpera et al., 2019b; Klicpera et al., 2019a] proposes to aggregate information from a larger (multi-hop) neighborhood at each layer, by sparsifying a generalized form of graph diffusion. This idea was also explored in [Liao et al., 2019; Luan et al., 2019; Xhonneux et al., 2020; Klicpera et al., 2019a] for multi-scale Graph Convolutional Networks. However, these methods do not incorporate attention mechanism which is crucial to model performance, and do not make use of edge embeddings (e.g., Knowledge graph) [Klicpera et al., 2019b]. Our approach defines a novel multi-hop context-dependent self-attention GNN which resolves the over-smoothing issue of GAT architectures [Wang et al., 2019a]. [Isufi et al., 2020; Cucurull et al., 2018; Feng et al., 2019] also extends attention mechanism for multihop information aggregation, but they require different set of parameters to compute the attention to neighbors of different hops, making these approaches much more expensive compared to MAGNA, and were not extended to the knowledge graph settings.

\section{Conclusion}

We proposed Multi-hop Attention Graph Neural Network (MAGNA), which brings together benefits of graph atten- tion and diffusion techniques in a single layer through attention diffusion, layer normalization and deep aggregation. MAGNA enables context-dependent attention between any pair of nodes in the graph in a single layer, enhances largescale structural information, and learns more informative attention distribution. MAGNA improves over all state-of-theart methods on the standard tasks of node classification and knowledge graph completion.

\section{References}

[Ba et al., 2016] Jimmy Lei Ba, Jamie Ryan Kiros, and Geoffrey E Hinton. Layer normalization. arXiv preprint arXiv:1607.06450, 2016.

[Bahdanau et al., 2015] Dzmitry Bahdanau, Kyunghyun Cho, and Yoshua Bengio. Neural machine translation by jointly learning to align and translate. In ICLR, 2015.

[Balazevic et al., 2019] Ivana Balazevic, Carl Allen, and Timothy Hospedales. Tucker: Tensor factorization for knowledge graph completion. In EMNLP, 2019.

[Bansal et al., 2019] Trapit Bansal, Da-Cheng Juan, Sujith Ravi, and Andrew McCallum. A2n: Attending to neighbors for knowledge graph inference. In $A C L, 2019$.

[Battaglia et al., 2018] Peter W Battaglia, Jessica B Hamrick, Victor Bapst, Alvaro Sanchez-Gonzalez, Vinicius Zambaldi, et al. Relational inductive biases, deep learning, and graph networks. arXiv preprint arXiv:1806.01261, 2018.

[Bordes et al., 2013] Antoine Bordes, Nicolas Usunier, Alberto Garcia-Duran, Jason Weston, and Oksana Yakhnenko. Translating embeddings for modeling multi-relational data. In NeurIPS, 2013.

[Chami et al., 2020] Ines Chami, Adva Wolf, Da-Cheng Juan, Frederic Sala, Sujith Ravi, and Christopher Ré. Low-dimensional hyperbolic knowledge graph embeddings. In ACL, 2020.

[Cucurull et al., 2018] Guillem Cucurull, Konrad Wagstyl, Arantxa Casanova, Petar Veličković, Estrid Jakobsen, Michal Drozdzal, Adriana Romero, Alan Evans, and Yoshua Bengio. Convolutional neural networks for mesh-based parcellation of the cerebral cortex. In $M I D L, 2018$.

[Defferrard et al., 2016] Michaël Defferrard, Xavier Bresson, and Pierre Vandergheynst. Convolutional neural networks on graphs with fast localized spectral filtering. In NeurIPS, 2016.

[Dettmers et al., 2018] Tim Dettmers, Pasquale Minervini, Pontus Stenetorp, and Sebastian Riedel. Convolutional 2d knowledge graph embeddings. In $A A A I, 2018$.

[Devlin et al., 2019] Jacob Devlin, Ming-Wei Chang, Kenton Lee, and Kristina Toutanova. Bert: Pre-training of deep bidirectional transformers for language understanding. In NAACL, 2019.

[Feng et al., 2019] Chenyuan Feng, Zuozhu Liu, Shaowei Lin, and Tony QS Quek. Attention-based graph convolutional network for recommendation system. In ICASSP, 2019.

[Gao and Ji, 2019] Hongyang Gao and Shuiwang Ji. Graph u-nets. In ICML, 2019.

[Gao et al., 2018] Hongyang Gao, Zhengyang Wang, and Shuiwang Ji. Large-scale learnable graph convolutional networks. In $K D D, 2018$.

[Hamilton et al., 2017] Will Hamilton, Zhitao Ying, and Jure Leskovec. Inductive representation learning on large graphs. In NeurIPS, 2017. 
[Isufi et al., 2020] Elvin Isufi, Fernando Gama, and Alejandro Ribeiro. Edgenets: Edge varying graph neural networks. arXiv preprint arXiv:2001.07620, 2020.

[Kipf and Welling, 2016] Thomas N Kipf and Max Welling. Semisupervised classification with graph convolutional networks. In ICLR, 2016.

[Klicpera et al., 2019a] Johannes Klicpera, Aleksandar Bojchevski, and Stephan Günnemann. Predict then propagate: Graph neural networks meet personalized pagerank. In ICLR, 2019.

[Klicpera et al., 2019b] Johannes Klicpera, Stefan Weißenberger, and Stephan Günnemann. Diffusion improves graph learning. In NeurIPS, 2019.

[Li et al., 2018] Qimai Li, Zhichao Han, and Xiao-Ming Wu. Deeper insights into graph convolutional networks for semisupervised learning. In $A A A I, 2018$.

[Liao et al., 2019] Renjie Liao, Zhizhen Zhao, Raquel Urtasun, and Richard S. Zemel. Lanczosnet: Multi-scale deep graph convolutional networks. In ICLR, 2019.

[Liu et al., 2019] Ziqi Liu, Chaochao Chen, Longfei Li, Jun Zhou, Xiaolong Li, Le Song, and Yuan Qi. Geniepath: Graph neural networks with adaptive receptive paths. In $A A A I, 2019$.

[Liu et al., 2020] Meng Liu, Hongyang Gao, and Shuiwang Ji. Towards deeper graph neural networks. In KDD, 2020.

[Lofgren, 2015] Peter Lofgren. Efficient Algorithms for Personalized PageRank. PhD thesis, Stanford University, 2015.

[Luan et al., 2019] Sitao Luan, Mingde Zhao, Xiao-Wen Chang, and Doina Precup. Break the ceiling: Stronger multi-scale deep graph convolutional networks. In NeurIPS, 2019.

[Ng et al., 2002] Andrew Y Ng, Michael I Jordan, and Yair Weiss. On spectral clustering: Analysis and an algorithm. In NeurIPS, 2002.

[Oono and Suzuki, 2020] Kenta Oono and Taiji Suzuki. Graph neural networks exponentially lose expressive power for node classification. In ICLR, 2020.

[Sandryhaila and Moura, 2013] Aliaksei Sandryhaila and José MF Moura. Discrete signal processing on graphs: Graph fourier transform. In ICASSP, 2013.

[Schlichtkrull et al., 2018] Michael Schlichtkrull, Thomas N Kipf, Peter Bloem, Rianne Van Den Berg, Ivan Titov, and Max Welling. Modeling relational data with graph convolutional networks. In ESWC, 2018.

[Sen et al., 2008] Prithviraj Sen, Galileo Namata, Mustafa Bilgic, Lise Getoor, Brian Galligher, and Tina Eliassi-Rad. Collective classification in network data. AI magazine, pages 93-106, 2008.

[Shang et al., 2019] Chao Shang, Yun Tang, Jing Huang, Jinbo Bi, Xiaodong He, and Bowen Zhou. End-to-end structure-aware convolutional networks for knowledge base completion. In $A A A I$, 2019.

[Shanthamallu et al., 2020] Uday Shankar Shanthamallu, Jayaraman J Thiagarajan, and Andreas Spanias. A regularized attention mechanism for graph attention networks. In ICASSP, 2020.

[Sun et al., 2019] Zhiqing Sun, Zhi-Hong Deng, Jian-Yun Nie, and Jian Tang. Rotate: Knowledge graph embedding by relational rotation in complex space. In ICLR, 2019.

[Tang et al., 2020] Yun Tang, Jing Huang, Guangtao Wang, Xiaodong He, and Bowen Zhou. Orthogonal relation transforms with graph context modeling for knowledge graph embedding. In $A C L, 2020$.
[Thekumparampil et al., 2018] Kiran K Thekumparampil, Chong Wang, Sewoong Oh, and Li-Jia Li. Attention-based graph neural network for semi-supervised learning. arXiv preprint arXiv:1803.03735, 2018.

[Toutanova and Chen, 2015] Kristina Toutanova and Danqi Chen. Observed versus latent features for knowledge base and text inference. In CVSC-WS, 2015.

[Trouillon et al., 2016] Théo Trouillon, Johannes Welbl, Sebastian Riedel, Éric Gaussier, and Guillaume Bouchard. Complex embeddings for simple link prediction. In ICML, 2016.

[Vaswani et al., 2017] Ashish Vaswani, Noam Shazeer, Niki Parmar, Jakob Uszkoreit, Llion Jones, Aidan N Gomez, Łukasz Kaiser, and Illia Polosukhin. Attention is all you need. In NeurIPS, 2017.

[Veličković et al., 2018] Petar Veličković, Guillem Cucurull, Arantxa Casanova, Adriana Romero, Pietro Lio, and Yoshua Bengio. Graph attention networks. In ICLR, 2018.

[Wang et al., 2019a] Guangtao Wang, Rex Ying, Jing Huang, and Jure Leskovec. Improving graph attention networks with large margin-based constraints. In NeurIPS-WS, 2019.

[Wang et al., 2019b] Quan Wang, Pingping Huang, Haifeng Wang, Songtai Dai, Wenbin Jiang, et al. Coke: Contextualized knowledge graph embedding. arXiv preprint arXiv:1911.02168, 2019.

[Weihua Hu, 2020] Marinka Zitnik Yuxiao Dong Hongyu Ren Bowen Liu Michele Catasta Jure Leskovec Weihua $\mathrm{Hu}$, Matthias Fey. Open graph benchmark: Datasets for machine learning on graphs. In NeurIPS, 2020.

[Wu et al., 2020] Zonghan Wu, Shirui Pan, Fengwen Chen, Guodong Long, Chengqi Zhang, and Philip S Yu. A comprehensive survey on graph neural networks. IEEE Trans Neural Netw Learn Syst, 2020.

[Xhonneux et al., 2020] Louis-Pascal A. C. Xhonneux, Meng Qu, and Jian Tang. Continuous graph neural networks. In ICML, 2020 .

[Xiong et al., 2020] Ruibin Xiong, Yunchang Yang, Di He, Kai Zheng, Shuxin Zheng, Chen Xing, Huishuai Zhang, Yanyan Lan, Liwei Wang, and Tie-Yan Liu. On layer normalization in the transformer architecture. In ICML, 2020.

[Xu et al., 2018] Keyulu Xu, Chengtao Li, Yonglong Tian, Tomohiro Sonobe, Ken-ichi Kawarabayashi, and Stefanie Jegelka. Representation learning on graphs with jumping knowledge networks. In ICML, 2018.

[Yang et al., 2015] Bishan Yang, Wen-tau Yih, Xiaodong He, Jianfeng Gao, and Li Deng. Embedding entities and relations for learning and inference in knowledge bases. In ICLR, 2015.

[Zhang et al., 2018] Jiani Zhang, Xingjian Shi, Junyuan Xie, Hao Ma, Irwin King, and Dit-Yan Yeung. Gaan: Gated attention networks for learning on large and spatiotemporal graphs. In $U A I$, 2018.

[Zhang et al., 2019] Shuai Zhang, Yi Tay, Lina Yao, and Qi Liu. Quaternion knowledge graph embedding. In NeurIPS, 2019.

[Zhang et al., 2020] Yongqi Zhang, Quanming Yao, Wenyuan Dai, and Lei Chen. Autosf: Searching scoring functions for knowledge graph embedding. In ICDE, 2020.

[Zhuang and Ma, 2018] Chenyi Zhuang and Qiang Ma. Dual graph convolutional networks for graph-based semi-supervised classification. In $W W W, 2018$. 